\title{
DIFICULDADES DE PROFISSIONAIS NA ATENÇÃO À SAÚDE DA PESSOA COM SURDEZ SEVERA ${ }^{1}$
}

\author{
PROFESSIONAL DIFFICULTIES IN HEALTH CARE OF \\ PATIENTS WITH SEVERE DEAFNESS
}

\section{DIFICULTADES PROFESIONALES EN LA ATENCIÓN EN SALUD A LA PERSONA CON SORDERA SEVERA}

\author{
Eurípedes Gil de França* \\ Maiary Andrade Pontes ${ }^{* *}$ \\ Gabriela Maria Cavalcanti Costa ${ }^{* * *}$ \\ Inácia Sátiro Xavier de França ${ }^{* * * *}$
}

\section{RESUMO}

Objetivo: Investigar as dificuldades de profissionais da saúde para a realização da consulta com a pessoa com surdez severa. Materiais e métodos: Estudo transversal, descritivo e qualitativo, realizado em março de 2011, na rede de atenção primária em saúde de Campina Grande - Paraíba. Utilizou-se um questionário estruturado, e 89 profissionais da saúde da Estratégia de Saúde da Família responderam sobre dificuldades para a consulta ao surdo. As respostas foram categorizadas por meio de Análise de Conteúdo na Modalidade Temática. Resultados: Dentre as dificuldades, destacaram-se: comunicação prejudicada, déficit na formação de recursos humanos para a consulta e reconhecimento das necessidades de saúde, infraestrutura inadequada para acolhimento e atendimento ao surdo, incerteza com relação aos cuidados em saúde prescritos na consulta e prejuízo da autonomia do paciente. Conclusão: Percebeu-se que a comunicação prejudicada constitui uma barreira para a promoção de saúde e que profissionais e unidades de saúde não estão capacitados para acolher e atender às necessidades de saúde da pessoa com surdez severa.

Palavras chave: Surdez, pessoal de saúde, atenção à saúde, barreiras de comunicação, enfermagem.

\begin{abstract}
Objective: To investigate the difficulties for health care professionals when dealing with patients with severe deafness in a medical appointment. Method: This is a cross-sectional, descriptive and qualitative study conducted in March 2011 in the Primary Health Care Network of Campina Grande - Paraíba. We used a structured questionnaire completed by 89 health professionals working in the Health Family Strategy. They

\footnotetext{
${ }^{1}$ Artigo extraído da Dissertação de Mestrado intitulada: Atenção à saúde do surdo na perspectiva do profissional da saúde. Programa de Pós-Graduação em Saúde Pública da Universidade Estadual da Paraíba, Campina Grande, Brasil.

*Enfermeiro. Doutorando. Universidade Estadual da Paraíba. Campina Grande, Paraíba, Brasil. Brasil. E-mail: euripedesgf@ gmail.com

"Enfermeira. Universidade Estadual da Paraíba. Campina Grande, Paraíba, Brasil. E-mail: maiary.uepb@hotmail.com

***Enfermeira. Professora Doutora. Universidade Estadual da Paraíba. Campina Grande, Paraíba, Brasil E-mail: gabymcc@ bol.com.br

${ }^{x * * *}$ Enfermeira. Professora Doutora. Universidade Estadual da Paraíba. Bolsista de Produtividade CNPq. Campina Grande, Paraíba, Brasil. E-mail: inacia.satiro@gmail.co
} 
were asked about the difficulties when dealing with deaf patients in a medical appointment. Their answers were categorized by means of a Thematic Content Analysis. Results: Among the main difficulties we find: impaired communication, a lack of trained human resources for these appointments and an insufficient recognition of these health needs, inappropriate infrastructure for deaf patient reception and care provision, uncertainty regarding the quality of the care provided at the medical appointment and the patient's lack of autonomy. Conclusions: We found that an impaired communication is a barrier to health promotion and that both health professionals and health units are not able to receive and meet the health needs of a patient with severe deafness.

Key words: Deafness, health personnel, health care, communication barriers, nursing.

\section{RESUMEN}

Objetivo: Investigar las dificultades de los profesionales de salud para la realización de la consulta con la persona con sordera severa. Materiales y métodos: Estudio transversal, descriptivo y cualitativo, realizado en marzo de 2011, en la red de atención primaria de salud de Campina Grande - Paraíba. Se utilizó un cuestionario estructurado, y 89 profesionales de salud de la Estrategia de Salud de la Familia respondieron sobre las dificultades para la consulta con el sordo. Las respuestas fueron categorizadas a través del Análisis de Contenido en la Modalidad Temática. Resultados: Entre las dificultades, se destacaron: comunicación perjudicada, déficit en la formación de recursos humanos para la consulta y reconocimiento de las necesidades de salud, infraestructura inadecuada para acoger y atender al sordo, incertidumbre con respecto a la atención sanitaria prescrita en la consulta y perjuicio de la autonomía del paciente. Conclusiones: Se observó que el déficit en la comunicación constituye una barrera para la promoción de la salud y que profesionales y unidades de salud no están cualificados para acoger y atender a las necesidades de salud de la persona con sordera severa.

Palabras clave: Sordera, personal de salud, atención a la salud, barreras de comunicación, enfermería.

Fecha recepción: 10/04/15 Fecha aceptación: 30/11/16

\section{INTRODUÇÃO}

A atenção à saúde da pessoa com deficiência $(\mathrm{PcD})$ diferencia-se entre aquilo que é proposto e o que existe na prática cotidiana dos serviços de saúde, visto que a sociedade parece não estar preparada para atender integralmente às necessidades de saúde desse público, resultando em dificuldades para a prática da inclusão social, acessibilidade e agravamento da deficiência. Diante disto, discutese a importância de se estabelecer estratégias que envolvam a família, a comunidade, serviços e profissionais da saúde, como meio de integralizar a assistência às necessidades das PcDs (1).

Discutir ressocialização e autonomia sig- nifica refletir inicialmente sobre a qualidade de vida, assistência à saúde da $\mathrm{PcD}$ e como estas impactam na vida desses usuários (2, 3). Mesmo considerando os avanços da ciência, dos sistemas de informação e tecnologias no processamento de dados, as informações quanto às reais necessidades de saúde das $\mathrm{PcD}$ são incompletas, resumindo-se a dados gerais (como aspectos sociodemográficos).

Nesse contexto, percebe-se ainda a falta de dados que evidenciem problemas de saúde de ordem biopsicossocial, discriminação e o preconceito, que dificultam tanto a empregabilidade quanto as condições propícias para o desenvolvimento de atividades laborais (4), além de casos de violência sexual e doméstica (5).

Com o intuito de deter o desenvolvimen- 
to de doenças crônicas e investir na qualidade da atenção e da assistência aos pacientes, o Ministério da Saúde do Brasil ampliou a Política Nacional de Promoção da Saúde (PNPS) como forma de alcançar todos os públicos e considerando suas necessidades de saúde. A Estratégia de Saúde da Família (ESF) apresenta-se como método utilizado para consolidação da Atenção Primária, mas somente com as novas prioridades em saúde possibilitou-se o alcance de populações com deficiência. A PNPS objetiva, por meio das unidades de saúde, diagnosticar agravos em saúde, tratá-los em nível primário, educar a população e prevenir novos casos (6).

As inadequações para acessibilidade e consulta a PcDs são relatadas na literatura e estão relacionadas ao déficit locomotor dessas pessoas, à falta de conhecimento dos profissionais da saúde em atender às necessidades específicas das $\mathrm{PcD}$, às barreiras físicas nas unidades de saúde e atitudinais, bem como à falta do conhecimento do potencial das pessoas com deficiência $(4,7)$. Nesse sentido, ainda que se considerem políticas, leis e decretos que garantam direitos nos cuidados em saúde e acessibilidade às $\mathrm{PcD}$, este grupo populacional possui restrições no que se refere ao usufruir dos benefícios garantidos nas legislações (7).

Dentre as deficiências, a auditiva acomete o usuário de três formas: surdez bilateral, parcial ou total (esta considerada também surdez severa ou profunda) (8). Usuários do terceiro nível, também chamados de surdos - ou pessoa com surdez severa (PcSS) - apresentam capacidade de escutar que varia desde a diminuição severa à perda total da audição, verificada por meio de exame de audiometria. Dentre os tipos de deficiência, a surdez severa destaca-se especialmente em relação à dificuldade em promover interação social, e esta se reflete no dia a dia como resultante da impossibilidade de estabelecer comunicação interpessoal $(9,10)$.

No contexto da comunicação, a limitação auditiva restringe as relações interpessoais, afetando também o acesso da PcSS aos serviços de saúde. A Unidade Básica de Saúde da Família (UBSF) atua como porta de entrada dos serviços de saúde; entretanto, há relatos (11) da precariedade no acolhimento às pessoas com limitação físico-sensorial visto à inacessibilidade aos ambientes e o despreparo profissional.

Em se tratando disso, evidencia-se (11) que profissionais da saúde não possuem capacitação adequada para comunicar-se com a PcSS, resultando em déficit na qualidade da consulta e grau de satisfação do usuário. Verificou-se também que as PcSS possuem necessidades de saúde que vão além da deficiência, mas não são diagnosticadas devido à falta de preparo do profissional em estabelecer um elo de entendimento.

Devido à incipiência das pesquisas em relação às consultas de saúde às $\mathrm{PcDs}(11)$, pouco se discute sobre a interação entre o profissional da saúde e a PcSS, a percepção do profissional e sua satisfação com o atendimento prestado. Investigar a opinião de profissionais com relação a esse tipo de assistência, conforme suas experiências assistenciais no dia a dia, no âmbito do Sistema Único de Saúde, possibilitará auxílio para o fomento de subsídios com fins de melhoria na formação e aperfeiçoamento de recursos humanos em saúde, em especial na área de atuação em questão.

Diante do exposto, questionou-se: quais dificuldades os profissionais da saúde enfrentam durante a consulta à PcSS? Para responder a esse questionamento, o presente estudo teve como objetivo investigar as dificuldades de profissionais da saúde para a realização da consulta à pessoa com surdez severa.

\section{MÉTODO}

Estudo descritivo e transversal. O presente estudo é uma análise qualitativa de parte de dados originais da Dissertação de Mestra- 
do intitulada: "Atenção à saúde do surdo na perspectiva do profissional da saúde". A pesquisa foi realizada em Campina Grande - Paraíba, em março de 2011, em 63 unidades de atenção primária à saúde da população.

Os dados foram coletados com 89 profissionais de saúde de nível superior, sendo 36 enfermeiros, 27 médicos, 18 cirurgiões dentistas, 5 assistentes sociais e, por fim, 1 fisioterapeuta, 1 psicóloga e 1 fonoaudióloga. Estes, em sua prática clínica, já haviam consultado uma PcSS. Logo, estavam aptos a responder o seguinte questionamento: em sua opinião, considerando sua última consulta a um paciente com surdez severa, quais foram as dificuldades encontradas para realização da consulta, considerando o atendimento em si?

Há de se registrar que, por se tratar de um recorte, este estudo não adotou qualquer critério para dimensionar a amostra qualitativa, a não ser o reconhecimento de experiência clínica declarada pelos profissionais.

Adotou-se como instrumento um formulário estruturado em quatro seções, que abordava respectivamente: dados de identificação, dados do atendimento ao surdo, dados sobre conhecimento de Libras (Língua Brasileira de Sinais) e, por fim, dados sobre capacitações em comunicação com surdo. Na seção dois deveriam falar sobre quais as dificuldades enfrentadas para o atendimento e quais as sugestões para minimizar as ditas dificuldades. Cada profissional respondeu individualmente ao instrumento de pesquisa, tendo sido, portanto, analisados 89 formulários.

Para a coleta de dados, os encontros foram agendados no serviço de saúde, em horário e dias previamente pactuados com os profissionais. De posse do material empírico, adotou-se como técnica de análise de dados a análise de conteúdo, na qual se faz o tratamento por inferências e interpretações. Segundo Minayo (12), diferentes são os tipos de análise de conteúdo: de expressão, das relações, de avaliação, de enunciação e categorial temática.
Para a autora, a categorização consiste no processo de redução do texto às palavras e expressões significativas, sendo necessário, portanto, seguir as etapas: 1) pré-análise, 2) exploração do material e 3) tratamento dos resultados, inferência e interpretação. Destarte, emergiram as categorias temáticas: "deficiência na formação de recursos humanos", "infraestrutura inadequada para o atendimento ao surdo", "incertezas quanto à promoção da saúde do surdo" e "restrição à autonomia do usuário".

Para cumprir o que determina a Resolução 466/2012, foi solicitada aos sujeitos a assinatura do Termo de Consentimento Livre e Esclarecido após compreenderem os objetivos da pesquisa e terem todas as informações sobre a garantia da não divulgação dos nomes e a confidencialidade das informações por elas oferecidas, além da preservação do direito de interromper a sua participação quando lhes fosse conveniente. A pesquisa foi aprovada pelo Comitê de Ética em Pesquisa da Universidade Estadual da Paraíba, conforme o CAAE no 0368.0.133.000-10.

\section{RESULTADOS}

\section{Categoria 1: Deficiência na formação de recursos humanos}

Os profissionais reconheceram que possuem déficit de conhecimentos para consultar a PcSS, bem como falta de preparo adequado para diagnosticar problemas de saúde e interagir com essa clientela. Informaram não terem recebido conhecimento específico na graduação que possibilitasse discussões e capacitação para o acolhimento e atendimento ao surdo. Considerando suas opiniões com relação à Gestão em Saúde da Família, alegaram que não há oferta de cursos de treinamento para o aprendizado de formas de comunicação com as PcSS. Essas ideias nitidamente são evidenciadas nos depoimentos: 
Falta de compreensão para poder passar melhor as orientações (Enfermeira 6).

Falta de dominio da linguagem Libras por minha parte (Enfermeira 19).

Falta de habilidade na comunicação, tendo em vista a não capacitação para lidar com o surdo (Enfermeira 34).

Não tenho habilidade em falar com o surdo, nem fui capacitado para tal (Médico 29).

\section{Categoria 2: Infraestrutura inadequada para $o$ atendimento ao surdo}

Registraram-se questões de infraestrutura inadequadas para o acolhimento e falta de recursos interativos para o auxílio durante a consulta. Os profissionais lamentam a inexistência de recursos audiovisuais que pudessem favorecer o entendimento da PcSS a respeito de suas queixas e como informá-las ao profissional. Segundo os participantes, não são todos os surdos que conseguem falar por Língua Brasileira de Sinais (Libras), portanto outros recursos para o uso da linguagem não verbal devem estar disponíveis:

Faltam materiais (brinquedos...) (Fonoaudió$\log a 1)$.

Falta de algo visual que colaborasse na comunicação com o usuário; também se houvesse um tempo maior para o atendimento destinado a esse usuário [...] Incapacidade na linguagem de sinais (Enfermeira 23).

\section{Categoria 3: Incertezas quanto à promoção da saúde do surdo}

Os profissionais citaram a comunicação prejudicada com a PcSS como principal problema para a realização da consulta, conforme evidenciado a seguir:

Dificuldade de entender e ser entendida (Enfermeira 8).

Quando o processo de fala e escuta não "ocorre", não há como haver uma boa comunicação (Enfermeira 16).
Dificuldade de comunicação e compreensão (Assistente social 2).

Não houve comunicação médico/paciente nem vice-versa, deixando este vínculo não estabelecido (Médica 32).

\section{Categoria 4: Restrição à autonomia do usuário}

Registrou-se que alguns profissionais solicitaram que o surdo retornasse à unidade acompanhado de um ajudante (familiar ou intérprete). Estes atuam como intermediadores, porém, por vezes, restringem a autonomia do surdo quando a consulta se dá mais entre profissional e acompanhante, como evidenciado a seguir:

Dificuldade de entender através de gestos, embora a comunicação tenha se dado mais com a mãe que a acompanhava (Enfermeira 13).

Paciente não fala, quem fez a consulta foi a mãe que estava acompanhando o paciente (Médica 5).

O fator comunicação foi totalmente comprometido já que não soube me comunicar ou repassar a informação clínica para o paciente, apenas para o seu acompanhante, algo frustrante, pois não sei como a informação chegou à mesma (Enfermeira 31).

O usuário atendido estava acompanhado, portanto é difícil dizer se os relatos do acompanhante corresponderam àquilo que o paciente realmente sente ou quer dizer (Médica 31).

\section{DISCUSSÃO E CONCLUSÃO}

A boa prática em saúde imprescinde do instrumento básico "Comunicação". Ao não utilizá-lo de forma eficaz e eficiente, corre-se o risco de a consulta apresentar lacunas, imprecisões ou incorreções. No caso específico da comunicação PcSS-profissional de saúde, o Estado brasileiro já tomou a iniciativa de legislar a respeito sancionando a Lei No 
10.436/ 2002, regulamentada pelo Decreto $5.626 / 2005$. Neste Decreto, consta que a rede de serviços do Sistema Único de Saúde (SUS) deve atender às pessoas surdas ou com deficiência auditiva, além de apoiar a formação e capacitação dos seus profissionais para o uso de Libras e sua tradução e interpretação (13).

No presente estudo, foi uma queixa comum a falta de capacitação em Libras, contribuindo com a dificuldade dos profissionais de saúde entrevistados em orientar melhor as PcSS. Contudo, acredita-se que a médio prazo essas queixas sejam minimizadas, dado que o ensino da Libras está se disseminando nos cursos de formação de recursos humanos para a saúde. Isto porque as Instituições de Ensino Superior já passaram a inserir no Projeto Pedagógico dos cursos da área de saúde e afins o componente Libras, possibilitando aos formandos a aquisição de habilidades e competências em Libras e para que a comunicação com a PcSS se desenvolva a contento.

Apesar dos participantes deste estudo revelarem falta de habilidade e de competência na comunicação com a PcSS, consoante relato de outros estudos (14), ao se afirmar essas limitações, os participantes deixam latente a necessidade de rever as próprias atitudes em relação à comunicação com essa pessoa, e de buscar a aquisição de recursos técnicos que possibilitem respeito e valorização dos direitos e desejos desse indivíduo.

No concernente às questões de infraestrutura inadequadas para o acolhimento e de falta de recursos interativos para o auxílio durante a consulta, urge inferir que estes aspectos se constituem barreiras de acessibilidade aos serviços de saúde, visto que a ausência de recursos técnicos que facilitem a comunicação da díade PcSS-profissional de saúde possibilita a ocorrência de vieses de interpretação, seja relacionados com as queixas da PcSS ou com as orientações do profissional.

Em se tratando da consulta de Enfermagem, autores (15) relatam que, no concer- nente a esse procedimento, o cuidar depende da interação entre cuidador e ser cuidado, e que as possíveis limitações durante a interação podem gerar entraves no processo comunicativo e falhas nas intervenções.

As propostas quanto à melhoria do processo de comunicação remetem prioritariamente à formação acadêmica. Observa-se, na literatura, que profissionais da saúde e da educação sentem a necessidade desse aprendizado. Verifica-se, em estudo (16), que os profissionais reconhecem os direitos da PcSS e concordam que o processo de inclusão social passa pela identidade individual, que no caso desses indivíduos trata-se de sua língua peculiar.

Considera-se, contudo, que a escassez de recursos materiais e a infraestrutura inadequada das unidades de saúde dificultam o processo de interação profissional-PcSS, considerando-se que, ainda que o profissional seja capacitado a comunicar-se por meios não verbais, nem toda PcSS possui o conhecimento formal da Libras. Dessa forma, e consoante outros autores (14), diferentes estratégias de comunicação devem estar disponíveis, como materiais ilustrativos, vídeo-aulas, cartazes, peças anatômicas e brinquedos que promovam essa interação.

Na perspectiva de melhorar a comunicação do profissional de saúde com o surdo, pesquisadores locados na academia capacitam os seus alunos por meio de leituras, debates e a utilização de vídeo nas vivências com participação de pessoas surdas. Para os autores, essa prática motivou os alunos a desenvolverem conhecimentos, habilidades de comunicação e atitudes positivas em relação a pacientes surdos (14).

Em se tratando da promoção da saúde da PcSS, também é preciso considerar que existem aquelas PcSS que só conseguem se comunicar por meio da linguagem oral e aquelas que só conseguem se expressar por meio da Libras. Daí porque, para além dos resultados do estudo atual, o surdo se considera em desvantagem perante o ouvinte e exemplifica 
essa condição com a dificuldade em compreender informações transmitidas em ambiente de trabalho, visto que costumam ser difundidas apenas pela modalidade oral (17).

Essas situações específicas da comunicação com a PcSS trazem implicações ao processo assistencial porque dificultam o empoderamento do indivíduo para tomar suas próprias decisões e usufruir os seus direitos de cidadania. Nesse sentido, autores alertam para o fato de que as atividades inerentes à promoção e prevenção em saúde auditiva ainda são muito restritas. Há, pois, que se lançar mão de capacitações dos profissionais de saúde atuantes nos processos de assistência à PcSS. Neste proceder, é preciso que o processo ensino e aprendizagem seja norteado por métodos motivadores, reflexivos e críticos para que os educandos sejam sujeitos ativos (18).

Outrossim, o aprendizado das formas de comunicação não verbal, mais que uma questão de saúde pública, remete à responsabilidade ética e social, pois o acolhimento da PcSS, por meio da compreensão das mensagens que elas emitem, resulta em inserção social e valorização do ser humano. Entendese, portanto, que o principal instrumento de trabalho do profissional passa a ser a comunicação (19).

Devido à inabilidade profissional em se comunicar por meio da Libras, uma prática comum de alguns profissionais consiste em solicitar que a PcSS se faça acompanhar por um familiar para intermediar o diálogo no decurso da consulta. Entende-se que a convivência parental permite ao acompanhante definir formas de comunicação, muitas vezes informais, porém eficazes quanto ao entendimento entre usuário e familiar, além do conhecimento do estilo de vida e das necessidades de saúde da PcSS.

A consulta em presença de acompanhante revela-se como estratégia para reduzir as possibilidades de diagnósticos e tratamentos errados, o que favorece diretamente a PcSS; no entanto, nega-se a esta pessoa o direito ao diálogo direto, comprometendo as ações de ressocialização deste indivíduo no âmbito da assistência à saúde. Percebe-se que, mesmo com acompanhantes ou intérpretes, o encontro entre profissionais e PcSS é permeado por barreiras de comunicação que comprometem o vínculo e a assistência (19).

A intermediação da comunicação pelo acompanhante tem implicações negativas para a PcSS, pois à medida que o acompanhante pergunta e responde por ela, torna-se notória a perda da sua autonomia. Uma vez cassado o seu direito de diálogo direto com o profissional, admite-se a sua exclusão da sociedade ouvinte. Também há que se pensar nas possíveis implicações éticas decorrentes de desrespeito à privacidade da PcSS e de quebra de sigilo acerca de agravos que ela não queira socializar com o acompanhante.

Em resposta à questão norteadora deste estudo, os participantes enfrentam dificuldades na consulta a PcSS devido à infraestrutura inadequada para o atendimento e à falta de capacitação em Libras. Dessa forma, a possibilidade de malefício, em decorrência da falta de comunicação eficaz, não apenas se reflete na saúde da PcSS, mas pode resultar em frustração para o profissional ao reconhecer a sua inaptidão para a resolução de problemas específicos do mundo da surdez.

Em meio às incertezas e demais sentimentos de impotência que permeiam a assistência à saúde da PcSS, frustração e insegurança apenas serão minimizadas ao passo que o profissional for inserido no mundo da surdez por meio da compreensão das particularidades desta cultura (16). Pensar em prejuízo unilateral (somente para o surdo) significa negligenciar as preocupações do profissional, desvalorizar seu esforço e culpá-lo pela incapacidade na resolução de problemas de saúde.

Mesmo existindo capital jurídico que regulamenta o atendimento às necessidades especiais da PcSS e fomenta o aperfeiçoamento profissional, os serviços de saúde e as instituições formadoras ainda têm dificuldade em 
atender às exigências ministeriais para o bom desempenho de tais ações. Talvez porque não perceberam, ainda, essa legislação como um incentivo a processos inovadores, ampliadores das chances de resolubilidade das ações em saúde para casos especiais como a surdez.

Necessita-se, portanto, de ações programadas entre gestão dos serviços, instituições de ensino, profissionais da saúde e comunidades, a fim de que os currículos atendam às necessidades prioritárias da cultura surda e a integralidade seja de fato exercida como direito do usuário nos cuidados à sua saúde. Mediante isto, os profissionais conseguirão desenvolver consultas mais eficazes e promover uma atenção integral como parâmetro fundamental para as estratégias em Atenção Primária.

Conclui-se que a falta de infraestrutura e de recursos adequados para o acolhimentos da PcSS resultam em estratégias improvisadas, informais, que restringem a identificação da real necessidade deste usuário. A dificuldade de interação profissional-PcSS compromete a prescrição segura de tratamentos, visto que a imprecisão na coleta de informações sobre o histórico de saúde do usuário gera incertezas. A presença de acompanhante reduz as possibilidades de erro, no entanto limita a autonomia da PcSS em decorrência da sua exclusão do processo de comunicação com o profissional.

Este estudo originou-se da preocupação de enfermeiros pesquisadores em relação a qualidade da consulta a PcSS. Emponderados, por meio de estudos desenvolvidos em grupo de pesquisa $(2-4,9-11,20)$, a respeito das dificuldades de pessoas com deficiência quanto ao acesso ao direito à integralidade na assistência à saúde, os autores desta pesquisa buscaram compreender as dificuldades de profissionais da saúde para a realização da consulta ao surdo.

Desta forma, esperamos contribuir incentivando os cursos de graduação a ampliarem as discussões a respeito da consulta a PcSS e incorporarem a disciplina de Libras na grade curricular. Esperamos, também, contribuir para a melhoria do processo de integralização da PcSS nos serviços e capacitar novos enfermeiros para uma assistência mais generalista, que inclua o surdo, servindo de inspiração para que outros cursos da área de saúde adotem a mesma estratégia na formação de recursos humanos.

\section{REFERÊNCIAS}

1. Cruz DM, Nascimento LRS, Vieira da Silva DMG, Dornelles Schoeller S. Rede de apoio à pessoa com deficiência física. Cienc. enferm. 2015; XXI(1): 23-33.

2. França ISX, Coura AS, França EG, Basílio NNV, Souto RQ. Quality of life of adults with spinal cord injuri: a study using the WHOQOL-bref. Rev Esc Enferm USP. 2011; 45(6): 1361-8.

3. Falcão SC, França ISX. Morbidity and mortality in disabled persons of the social security in Brazil. Cien Saude Colet. 2016; 15(2): 242-9.

4. Aragão JS, França ISX, Coura AS, Medeiros CCM, Enders BC. Vulnerability associated with sexually transmitted infections in physically disabled people. Cien Saude Colet. 2016; 21(10): 3143-52.

5. Lee K, Devine A, Marco J, Zayas J, GillAtkinson L, Vaughan C. Sexual and reproductive health service for women with disability: a qualitative study with service providers in the Philippines. BMC Womens Health. [Internet]. 2015 Oct. [citado 07 nov 2016]; 15(87). Disponível em: ht t p:// b m cwomenshealth. biomedcentral.com/articles/10.1186/ s12905-015-0244-8

6. UNA-SUS [Internet]. Brasil: Ministério da Saúde, Secretaria de Gestão do Trabalho e da Educação em Saúde, UNA-SUS. Ministério lança Política Nacional de Promoção da Saúde; 2014 Nov 3 [citado 03 nov 2016]. Disponível 
em: http://www.unasus.gov.br/noticia/ ministerio-lanca-politica-nacional-depromocao-da-saude

7. Pereira PEC, Caldas ASC, Cabral AKPS. Inclusão profissional de pessoas com deficiência física em um serviço de saúde do Estado de Pernambuco. Rev Ter Ocup Univ São Paulo. 2016; 27(2): 146-55.

8. Presidência Da República, Casa Civil, Subchefia para Assuntos Jurídicos (BR). Decreto no 5.296 de 02 de dezembro de 2004. Regulamenta as Leis no 10.048 , de 8 de novembro de 2000 , que dá prioridade de atendimento às pessoas que especifica, e 10.098 , de 19 de dezembro de 2000, que estabelece normas gerais e critérios básicos para a promoção da acessibilidade das pessoas portadoras de deficiência ou com mobilidade reduzida, e dá outras providências. Portal da Legislação. Decretos. 2004. [citado 27 nov 2016]. Disponível em: http://www.planalto.gov.br/ ccivil_03/_ato2004-2006/2004/decreto/ D5296.htm

9. Aragão JS, Magalhães IMO, Coura AS, Silva AFR, Cruz GKP, França ISX. Access and communication of deaf adults: a voice silenced in health services. J. res.: fundam. care online [Internet]. 2014 Jan-Mar [citado 27 nov 2016]; 6(1): 1-7. Disponível em: http://www.seer.unirio. $\mathrm{br} /$ index.php/cuidadofundamental/ article/view/2989/pdf_1091

10. Oliveira YCA, Celino SDM, França ISX, Pagliuca LMF, Costa GMC. Conhecimento e fonte de informações de pessoas surdas sobre saúde e doença. Interface. 2015; 19(54): 549-60.

11. Oliveria YCA, Coura AS, Costa GMC, França ISX. Communication between health professionals-deaf people. J Nurs UFPE on line [Internet]. 2014 Feb. [citado 27 nov 2016]; 9(Suppl. 2): 957-64. Disponível em: http://www.revista.ufpe. br/revistaenfermagem/index.php/revista/article/view/5502/pdf_7317

12. Minayo MCS. O desafio do conhecimen- to: pesquisa qualitativa em saúde. 5. ed. São Paulo: Hucitec-Abrasco, 1998. 269 p. 13. Presidência Da República, Casa Civil, Subchefia para Assuntos Jurídicos (BR). Decreto no 5.626, de 22 de dezembro de 2005. Regulamenta a Lei no 10.436 , de 24 de abril de 2002, que dispõe sobre a Língua Brasileira de Sinais - Libras, e o art. 18 da Lei no 10.098 , de 19 de dezembro de 2000. Diário Oficial da República Federativa do Brasil. 2005. [citado 27 nov 2016]. Disponível em: http://www.planalto.gov. br/ccivil_03/_ato2004-2006/2005/decreto/d5626.htm

14. Costa LSM, Silva NCZ. Desenvolvendo atitudes, conhecimentos e habilidades dos estudantes de medicina na atenção em saúde de pessoas surdas. Interface. 2012; 16(43): 1107-17.

15. Trigueiro Filho EPS, Silva JPG, Freitas FFQ, Alves SRP, Costa KNFM. Perception of nursing students on the communication with people with visual and hearing disabilities. J Nurs UFPE on line [Internet]. 2013 [citado 27 nov 2016]; 7(1): 747-54. Disponível em: http://www.revista.ufpe.br/revistaenfermagem/index. php/revista/article/view/3577/pdf_2169

16. Machado WCA, Figueiredo NMA, Tonini T, Silva CRL, Silva RCL. The search for a brazilian sing course: a descriptive exploratory study. Online braz $\mathrm{j}$ nurs [Internet]. 2012. [citado 25 julio 2013]; 11(2): [10 p]. Disponível em: http://www.objnursing.uff.br/index.php/nursing/article/view/3664/html

17. Lopes MAC, Leite LP. Concepções de surdez: a visão do surdo que se comunica em língua de sinais. Rev. Bras. Ed. Esp., Marília [Internet]. 2011 Mai-Ago. [citado 27 nov 2016]; 17(2): 305-20. Disponível em: http://portal.sme.prefeitura.sp.gov. br/Portals/1/Files/19774.pdf

18. Ribeiro GM, Figueiredo MFS, Rossi-Barbosa LAR. A importância da capacitação em saúde auditiva: uma revisão integrativa. Rev. CEFAC. [Internet]. 2014 Jul-A- 
go. [citado 27 nov. 2016]; 16(4): 131825. Disponível em: http://www.scielo. br/scielo.php?script=sci_arttext\&pi$\mathrm{d}=$ S1516-18462014000401318

19. Bentes IMS, Vidal ECF, Maia ER. Deaf person's perception on health care in a midsize city: an descriptive-exploratory study. Online braz j nurs [Internet]. 2011 [citado 25 jul 2013]; 10(1): [10 p]. Disponível em: http://www.objnursing.uff.br/index.php/nursing/article/ view/j.1676-4285.2011.3210.2

20. França ISX, Coura AS, França EG, Cavalcante GMC, Sousa FS. Application of principlist bioethics to public policies for disabled people: systematic review. Online braz j nurs [Internet]. 2010 [citado 21 mar 2017]; 9(1) [14 p]. Disponível em: http://www.objnursing.uff.br/index.php/nursing/article/ view/j.1676-4285.2010.2893/653 